\title{
The influence of pro-longevity gene Gclc overexpression on the age-dependent changes in Drosophila transcriptome and biological functions
}

\author{
Alexey Moskalev 1,2,3,4*, Mikhail Shaposhnikov ${ }^{2,4}$, Ekaterina Proshkina ${ }^{2,4}$, Alexey Belyi ${ }^{2,4}$, Alexander Fedintsev ${ }^{3}$, \\ Svetlana Zhikrivetskaya', Zulfiya Guvatova', Asiya Sadritdinova', Anastasia Snezhkina', George Krasnov \\ and Anna Kudryavtseva ${ }^{{ }^{*}}$
}

From The International Conference on Bioinformatics of Genome Regulation and StructurelSystems Biology (BGRSISB-2016) Novosibirsk, Russia. 29 August-2 September 2016

\begin{abstract}
Background: Transcriptional changes that contribute to the organism's longevity and prevent the age-dependent decline of biological functions are not well understood. Here, we overexpressed pro-longevity gene encoding glutamate-cysteine ligase catalytic subunit (Gclc) and analyzed age-dependent changes in transcriptome that associated with the longevity, stress resistance, locomotor activity, circadian rhythmicity, and fertility.

Results: Here we reproduced the life extension effect of neuronal overexpression of the Gclc gene and investigated its influence on the age-depended dynamics of transcriptome and biological functions such as fecundity, spontaneous locomotor activity and circadian rhythmicity, as well as on the resistance to oxidative, proteotoxic and osmotic stresses. It was shown that Gclc overexpression reduces locomotor activity in the young and middle ages compared to control flies. Gclc overexpression slowed down the age-dependent decline of locomotor activity and circadian rhythmicity, and resistance to stress treatments. Gclc level demonstrated associations with the expression of genes involved in a variety of cellular processes including Jak-STAT, MAPK, FOXO, Notch, mTOR, TGF-beta signaling pathways, translation, protein processing in endoplasmic reticulum, proteasomal degradation, glycolysis, oxidative phosphorylation, apoptosis, regulation of circadian rhythms, differentiation of neurons, synaptic plasticity and transmission.
\end{abstract}

Conclusions: Our study revealed that Gclc overexpression induces transcriptional changes associated with the lifespan extension and uncovered pathways that may be associated with the age-dependent decline of biological functions.

Keywords: Glutathione, Lifespan, Gene expression, Locomotor activity, Circadian rhythmicity, Fertility, Drosophila melanogaster

\footnotetext{
* Correspondence: amoskalev@list.ru; rhizamoeba@mail.ru

${ }^{1}$ Engelhardt Institute of Molecular Biology, Russian Academy of Sciences,

Moscow, Russia

Full list of author information is available at the end of the article
} International License (http://creativecommons.org/licenses/by/4.0/), which permits unrestricted use, distribution, and reproduction in any medium, provided you give appropriate credit to the original author(s) and the source, provide a link to the Creative Commons license, and indicate if changes were made. The Creative Commons Public Domain Dedication waiver (http://creativecommons.org/publicdomain/zero/1.0/) applies to the data made available in this article, unless otherwise stated. 


\section{Background}

The stimulation of defense cellular systems leads to aging attenuation and lifespan extension in different organisms. For example, the enhancement of glutathione biosynthetic capability can determine longevity and delay aging. Previously, Dr. William Orr et al. demonstrated the pro-longevity role of glutamatecysteine ligase $(\mathrm{Gcl})$, which is a main catalyzer in the de novo glutathione synthesis [1]. Overexpression of genes encoding $\mathrm{Gcl}$ catalytic and modulatory subunits (Gclc and Gclm) was found to extend Drosophila lifespan and stimulate oxidative stress resistance without affecting the metabolic rate [1]. Additionally, previous studies have shown that the activity of $\mathrm{Gcl}$ and the amount of $\gamma$-glutamylcysteinylglycine $(\mathrm{GSH})$, the protein that it synthesise, related to stress resistance levels $[2,3]$.

This paper aims to reveal pathways involved in the organism's longevity and associated with the agedependent decline of biological functions and stress resistance. We analyzed the effects of neuronal overexpression of Gclc on the lifespan, resistance to oxidative, proteotoxic and osmotic stresses, age-dependent dynamics of locomotor activity, fecundity, and transcriptomic changes. We reproduced the life extension effect of neuronal overexpression of the Gclc gene and demonstrated that Gclc overexpression slows down the age-dependent decline of locomotor activity and circadian rhythmicity without effect on fecundity. Transcriptome analysis revealed pathways that may contribute to the longevity and prevent the age-dependent decline of biological functions.

\section{Methods \\ Drosophila melanogaster strains UAS-Gclc}

Carries an additional copy of the gene of catalytic subunit of glutamate-cysteine ligase $(\mathrm{Gclc})$ under the control of UAS promoter on the chromosome 2. Gclc catalyzes the rate-limiting reaction in the de novo glutathione biosynthesis. Kindly provided by Dr. W.C. Orr (Southern Methodist University, Dallas, USA) [1].

\section{Appl-GAL4}

Driver line containing GAL4 selectively expressed in nervous system cells (\#32040, Bloomington Drosophila Stock Center).

In order to match the genetic background of $U A S$ and GAL4 strains used in this study, flies were backcrossed into $w^{1118}$ (\#3605, Bloomington Drosophila Stock Center, USA) for 6-8 times.

\section{Activation of overexpression}

To activate the overexpression of the Gclc gene the GAL4-UAS system was used [4]. We used constitutively active neuronal driver Appl-GAL4 that activate the $U A S$ Gclc overexpression in Drosophila heads [1]. UAS-Gclc flies from the parental line were used as a control.

\section{Lifespan analysis}

The virgin females and males were used in the experiments. Animals were maintained in the Binder KBF720-ICH (Binder, Germany) climate chamber on the sugar-yeast medium at $25{ }^{\circ} \mathrm{C}$ in a $12 \mathrm{~h}$ light- $12 \mathrm{~h}$ dark regime and at $60 \%$ relative humidity. Three-five Drosophila vials (Genesee Scientific, USA) containing 30 flies per vial were used in each experiment replication. Experiments were performed in 3 replicates. A total of 350-450 males and 350-450 females were analyzed. Animals were relocated to a fresh medium two times a week. Dead flies were counted daily. The median and maximum (as the age of $90 \%$ mortality) lifespan the were evaluated. The Mantel-Cox test was used to estimate the statistical differences in the median lifespan between control and experimental groups. The Wang-Allison test was used to compare the statistical differences in the maximum lifespan [5]. Statistical analysis was carried out using $R$ ( $R$ core Team), version 2.15.1. The survival curves were plotted using STATISTICA, version 6.1 (StatSoft, USA).

\section{Sample collection and RNA isolation}

Transcriptomic analysis was performed using control UAS-Gclc flies and flies with Gclc overexpression at the age of 1 (young), 4 (matured) and 6 weeks (old). Forty males and females heads were prepared separately for each experimental variant in 3 replicates. Fly transcriptome is strongly gender-dependent and one should not pool flies with different sex into one group. To reveal age-dependent and Gclc-induced transcriptomic changes, we applied multivariate generalized linear models (GLM) analysis. Total RNA was isolated from imago heads using QIAzol Lysis Reagent (Qiagen, Netherlands) with the isopropanol precipitation. The concentration of RNA was assessed using Qubit ${ }^{\circ} 2.0$ Fluorometer (Invitrogen, USA) and NanoDrop ${ }^{\circ}$ ND1000 spectrophotometer (NanoDrop Technologies Inc., USA). The A260/A280 ratios of RNA samples were 1.82.0. The integrity of the isolated RNA (RIN) was measured with Bioanalyzer Agilent 2100 (Agilent Technologies, USA). RIN values for all samples didn't be less than 8.0. All samples were treated with DNase I (Fermentas, Lithuania).

\section{Library preparation and sequencing of mRNA}

Sample preparation and RNA sequencing were carried out by previously used protocol with modifications $[6,7]$. The Illumina TruSeq ${ }^{\mathrm{TM}}$ RNA Sample Preparation Kit (LowThroughput protocol) was used to prepare samples for mRNA sequencing libraries. 
To purify poly-A containing mRNA molecules from total RNA samples $(2.5 \mu \mathrm{g})$ the poly-T oligo-attached magnetic beads were used in two rounds of purification. During the second round of purification RNA was fragmented and primed for cDNA synthesis. Then the cDNA synthesis was performed using SuperScript Double-Stranded cDNA Synthesis Kit (Invitrogen, USA). cDNA was converted into the double-stranded (ds) cDNA. The ds cDNA was isolate from the secondstrand reaction mix using Ampure XP beads.

The end-repair reaction was used to create blunt ends on the ds cDNA. To avoid the ligation of blunt ends during the adapter ligation reaction, a single 'A' nucleotide was added to the $3^{\prime}$ ends of them. The specific RNA Adapter Indexes supplied in the kit were ligated to cDNA fragments. The In-Line Control DNA was added to each enzymatic reaction. The PCR process (15 cycles) was used to selectively enrich DNA fragments with adapter molecules on both ends and to amplify the amount of DNA in the library, according to the manufacturer's protocol.

The quantity of libraries was determined using the qPCR method by Rotor-Gene 6000 PCR System (Qiagen, USA) according to the manufacturer's protocol. Primers matched sequences within adapters flanking an Illumina sequencing library. Before starting qPCR, a control template was selected to measure the libraries for quantification. The quality of libraries was defined using Agilent 2100 Bioanalyzer (Agilent, USA) according to the manufacturer's protocol. The final product showed a band of approximately 260 base pairs.

cDNA libraries were normalized to $2 \mathrm{nM}$, pooled together in equal volumes, and sequenced with $50 \mathrm{bp}$ single-end reads on the HiSeq 2000 platform (Illumina, USA). Illumina HiSeq Analysis Software was used to obtain raw sequencing reads. The sequencing data were stored in FASTQ format. At least 25 million reads were obtained for each pool of flies.

\section{Sequencing data processing}

Processing of transcriptomic data was performed using PPLine toolkit [8] including read preprocessing (trimmomatic), mapping (STAR) and counting (HTSeq-count). The further analysis was done with $R$ programming language ( $\mathrm{R}$ core Team). The edgeR package was used for analysis of differential expression [9]: we used Student $t$ test to compare two groups and generalized linear models (GLM) for complex comparisons in order to reveal genotype-, sex- and gender-associated transcriptomic changes on complete sampling (model ' $\sim$ Age + Gender + Genotype). KEGG gene set enrichment analysis (GSEA) was performed using clusterProfiler [10]. To visualize altered KEGG pathways we modified pathview Bioconductor package [11] in order to take into account the absolute gene expression level, e.g. normalized read count per million (CPM) or FPKM. Aging is associated with global transcriptomic changes. Many KEGG nodes are mapped to multiple genes/proteins. For example, KEGG SdhA is related to both FBgn0261439/SdhA and FBgn0036222/ $S d h A L$. SdhAL is a minor isoform of succinate dehydrogenase; it is overexpressed during the aging $\left(\operatorname{LogFC} C_{\text {SdhAL }}\right.$ $=+5.2)$ and has the absolute expression level (CPM/ FPKM) 100-fold lesser than its major isoform SdhA which is down-regulated in aged flies $\left(\operatorname{LogFC} C_{S \mathrm{dhA}}=-2.1\right)$. An original pathview package summarizes LogFC within each KEGG node, and the final LogFC for succinate dehydrogenase is +3.1 , and this is not correct since minor $S d h A L$ does not play any significant role here. To avoid this trouble, we have modified the pathview package to calculate the final $\operatorname{LogFC}$ as weighted sum of $\operatorname{LogFC}$ for each component: $\operatorname{LogFC} C_{\text {final }}=\left(\operatorname{LogFC} \mathrm{CdhA}_{\mathrm{Sd}} \cdot \mathrm{CPM}_{\mathrm{SdhA}}+\right.$ $\left.\operatorname{LogFC} \mathrm{SdhAL}_{\mathrm{S}} \cdot \mathrm{CPM}_{\mathrm{SdhAL}}\right) /\left(\mathrm{CPM}_{\mathrm{SdhA}}+\mathrm{CPM}_{\mathrm{SdhAL}}\right)$. This correction is critical when analyzing and visualizing transcriptomic changes affecting hundreds genes.

\section{Stress resistance dynamic analysis}

To assess the changes in stress resistance of flies overexpressing the Gclc gene, 150 flies (30 flies per vial) were collected in each experimental variant at 10 different ages $-7,14,21,28,35,42,49,56,63,70$ days. Males and females were analyzed separately. Following stress treatments were used: oxidative $(20 \mathrm{mM}$ paraquat, $\left.15 \mathrm{mM} \mathrm{CuSO}_{4}\right)$, osmotic $(400 \mathrm{mM} \mathrm{NaCl})$ and proteotoxic stress $\left(5 \mathrm{mM} \mathrm{CdCl}_{2}\right)$. Flies were deprived of food and water for $3 \mathrm{~h}$ and were transferred into vials containing a filter paper moistened with $350 \mathrm{ml}$ of the $5 \%$ sucrose solution with one of the substances. Two times a day, we counted the number of dead animals. Flies were transferred into new vials every two days. Flies were kept under stress until the end of life. The mean, median survival time, and the time of $90 \%$ mortality were calculated.

We determined the dependence of survival on age based on the Pearson correlation coefficient. Additionally, we evaluated the impact of age, gender, genotype and the nature of stress factors on the survival of flies by using Cox proportional hazards models. In the Cox regression analysis, we assumed that risk factors are the age of flies in which they were subjected to stress impact and their genotype. To determine the hazard ratio of death for each fly when exposed to stress, we compared contributions of sex and age specimens at different stresses for UAS-Gclc and Appl-GAL4 > UAS-Gclc flies. Next, we compared the risk for flies of each experimental group with the interaction of factors - gender and genotype. Thus, we determined how age, sex, and genotype affected the risk of death. Data analysis was performed using the STATISTICA software, version 6.1 
(StatSoft, USA), OASIS [12], and $\mathrm{R}$ programming language (R Core Team).

\section{Analysis of fecundity and fertility}

Before the analysis, females with and without overexpression of the Gclc gene were maintained with young wild-type Canton-S males for mating during $24 \mathrm{~h}$. Mated females were put separately into the vials with a nutrient colored with activated carbon for egg-laying for $24 \mathrm{~h}$. The number of eggs laid by females (fecundity) and the number of imago developed from the eggs by the 10-15th day after egg laying (fertility) were counted. Fecundity and fertility were estimated once a week. Experiment was performed in 2 replicates (a total of 100 females per experimental variant). To compare statistical significance between flies overexpressing the Gclc gene and flies without overexpression, $\chi^{2}$ criterion was used.

\section{Locomotor activity analysis}

The age-dependent dynamics of spontaneous locomotor activity was analyzed using Drosophila Locomotor Activity Monitor (Trikinetics, USA). Locomotor activity of 1 , 4 , and 6-week-old flies was recorded. The data were collected during $24 \mathrm{~h}$ and represented as average locomotor activity in $10 \mathrm{~min}$ bins or average total daily locomotor activity. The average locomotor activity was calculated between three vials each containing 10 flies.

\section{Circadian rhythmicity analysis}

Locomotor activity of 5,30, and 50-day-old males was recorded for 2 days in $12 \mathrm{~h}$ light : $12 \mathrm{~h}$ darkness conditions (LD), followed by 6 days in constant darkness (DD) using Drosophila Activity Monitor (Trikinetics, USA). For the analysis and visualization of circadian rhythmicity the ActogramJ software package [13] based on ImageJ Image Analysis Software [14] was used.

\section{Results}

Lifespan

Overexpression of the Gclc gene led to the increase of median and maximum lifespan of Drosophila males and females compared with the control line UAS-Gclc (Table 1, Fig. 1). Thus, we reproduced the lifespan expending effect of Gclc overexpression, which was published earlier by Dr. William Orr et al. [1].

\section{Analysis of the transcriptome}

We derived RNA-Seq expression profiles for 9400 genes (after eliminating low expression ones). We revealed the greatest transcriptomic changes during Drosophila aging: 3326 genes $(p<0.05)$ demonstrated expression associations with age (1885 of them passed FDR $<0.05$ threshold), whereas alterations in $1442(p<0.05 ; 376$ of 1442 have FDR $<0.05)$ we found to be sex-specific. Flies with enhanced Gclc activity showed lesser effect on gene expression profiles: only 188 genes $(p<0.05)$ were found to be associated with Gclc overexpression using the GLM model ' Age + Gender + Genotype' ( $p<0.5 ; 28$ of 188 have FDR $<0.05$ ).

We performed GSEA based on GO annotation and pathway analysis based on KEGG data for differentially expressed genes that passed $p<0.05$ threshold (Fig. 2, Additional file 1). We found the reduction of mitochondrial function, oxidative phosphorylation, ribosome biogenesis and translation as one of the hallmarks of aging. On the other hand, we found the age-dependent upregulation of apoptosis pathways, Wnt, mTOR/PI3K, FOXO pathways, circadian rhythm genes, enhanced cAMP signaling, overexpression of Myc and Notch.

\section{Stress resistance}

We analyzed age-dependent changes of the resistance to different kinds of stress factors of flies with constitutive overexpression of the Gclc gene and the control line UAS-Gclc. Survival rates of flies under conditions of oxidative stress (paraquat, $\mathrm{CuSO}_{4}$ ), proteotoxic stress $\left(\mathrm{CdCl}_{2}\right)$ and osmotic stress $(\mathrm{NaCl})$ were estimated. Mean and median survival time, as well as the time of $90 \%$ mortality were decreased with age both in individuals with Gclc overexpression and in control flies under the treatment by all used stress factors (Table 2), that is discernible by the variation of survival curves (Figs. 3, 4, 5, and 6). Analysis using the Cox proportional hazard models also demonstrated that age is a risk factor in studied stress conditions for both genotypes (Table 3).

In order to compare the dynamics of survival of flies with and without overexpression of the Gclc gene, the

Table 1 Influence of Gclc overexpression on median and maximum lifespan (the results of 3 replicates are combined)

\begin{tabular}{|c|c|c|c|c|c|c|c|c|}
\hline Genotype & Sex & M (days) & $\mathrm{dM}$ & $\begin{array}{l}\text { Gehan-Wilcoxon test } \\
\text { (p-value) }\end{array}$ & $90 \%$ (days) & $d 90 \%$ & $\begin{array}{l}\text { Wang-Allison test } \\
\text { (p-value) }\end{array}$ & $n$ \\
\hline UAS-GClc (control) & males & 50 & & & 72 & & & 449 \\
\hline Appl-GAL4 > UAS-Gclc (overexpression) & males & 65 & $+23.1 \%$ & $p<0.0001(0)$ & 76 & $+5.3 \%$ & $p<0.001(0.00014)$ & 419 \\
\hline UAS-Gclc (control) & females & 53 & & & 72 & & & 464 \\
\hline Appl-GAL4 > UAS-Gclc (overexpression) & females & 76 & $+30.3 \%$ & $p<0.0001(0)$ & 88 & $+18.2 \%$ & $p<0.0001(0)$ & 390 \\
\hline
\end{tabular}

M - median lifespan, $90 \%$ - age of $90 \%$ mortality (maximum lifespan), dM - differences between median lifespan of control and Gclc overexpressed flies, d90\% differences between age of $90 \%$ mortality of control and Gclc overexpressed flies, $n$ - number of flies 


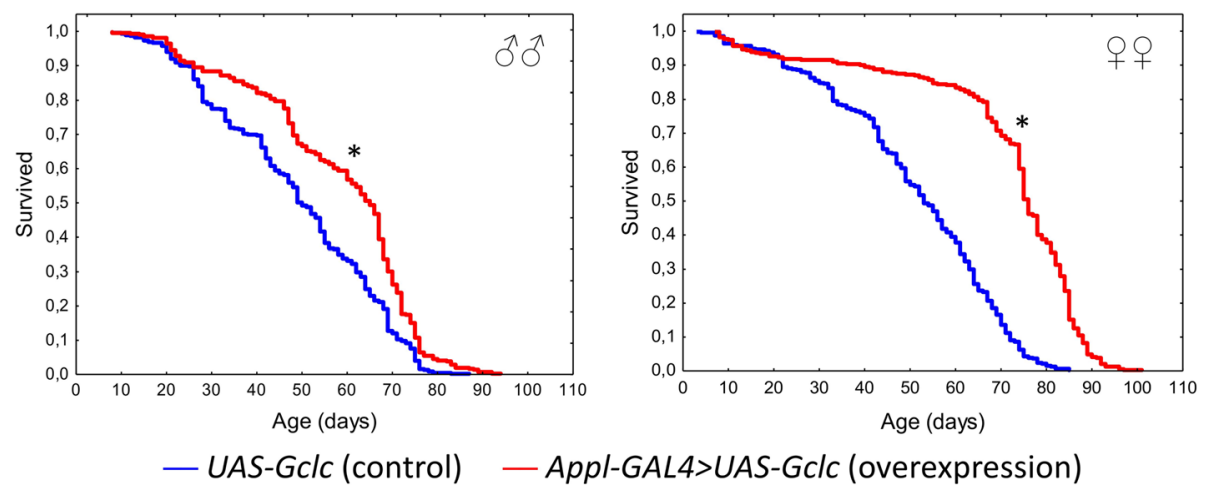

Fig. 1 Influence of Gclc overexpression on lifespan (the results of 3 replicates are combined). ${ }^{*} p<0.001$, Kolmogorov-Smirnov test

correlation analysis was performed using the Pearson's correlation coefficient. The correlation coefficient was calculated based on the mean survival time for all experimental variants at each age group (Table 4). A high negative correlation was revealed between survival and age in both males and females (Pearson's correlation coefficient $>0.9 ; p<0.001$ ). This is also evident by the large angle of inclination of regression lines scatterplots (Figs. 7 and 8).

To study age-dependent differences of stress resistance of Appl-GAL4 > UAS-Gclc flies and control UAS-Gclc flies, we calculated the value of the difference between the correlation coefficients (Table 5). In all cases, no significant differences in the age-dependent dynamics were found.

Clouds variable in the scatter diagrams and linear regression in flies overexpressing Gclc in all variants except the males under exposure by $\mathrm{NaCl}$ are placed higher compared to the control line, thus it is possible to note a higher survival rate in different age groups as compared to the control line (Figs. 7 and 8). The linear regression of males' survival under the influence of $\mathrm{NaCl}$ has similar values of the tilt angle and located close to each other. Thus, we can conclude that both flies with Gclc overexpression and without overexpression have similar levels of stress resistance.

Analysis using the Cox proportional hazards model demonstrated that under the influence of all examined stress males and females overexpressing Gclc have significantly lower risk of death compared with control line in all ages. Under the conditions of paraquat treatment, the stress tolerance of females overexpressing Gclc were not significantly differed from males, as well as UASGclc females were less resistant to stress than males. In this study, overexpression of Gclc gene didn't lead to significant changes in the dynamics of stress resistance. However, the previous work showed a higher resistance to oxidative stress in aged flies against a background of the increased activity of Gclc, and in young individuals stress resistance was on the level of control [1]. However, this effect was observed only in some embodiments of the experiment with a specific driver and dose of stress. Additionally, females with UAS-Gclc and ApplGAL4 > UAS-Gclc genotypes were more resistant to stress than males, in spite of used treatment (Table 3).

\section{Fecundity and fertility}

To estimate the age-dependent changes of fecundity and fertility, the number of eggs laid by females during $24 \mathrm{~h}$ was calculated every week during Drosophila lifetime, and the number of adult flies developed from these eggs was estimated after 10-15 days. We didn't found statistically signifying differences of these parameters between females with Gclc overexpression and without overexpression (Fig. 9). However, the tendency to the increase of the percentage of progeny developed to imago was displayed in flies with increased Gclc expression (Fig. 9).

Surprisingly, we found the increase of the activity of many genes participating in the reproductive function during aging, except for genes involved in vitellogenesis which are primarily down-regulated both in aged flies and flies with Gclc overexpression. The fecundity and fertility analysis revealed the reduction of these parameters in aged flies and tendency to increase the percentage of imagoes developed from eggs of flies with Gclc overexpression compared with control.

\section{Locomotor activity}

The dynamics of age-dependent changes of locomotor activity was estimated in flies at the age of 1, 4, 6 weeks. It was found that Gclc overexpression decreased the total daily activity of 4-week-old males and 1-week-old females (Fig. 10). At the same time, the daily activity of flies with Gclc overexpression didn't change during aging compared with control animals (Fig. 10).

The analysis of average daily locomotor activity of flies at different ages educed that the decrease of locomotor activity level of 1-week-old females with Gclc overexpression 


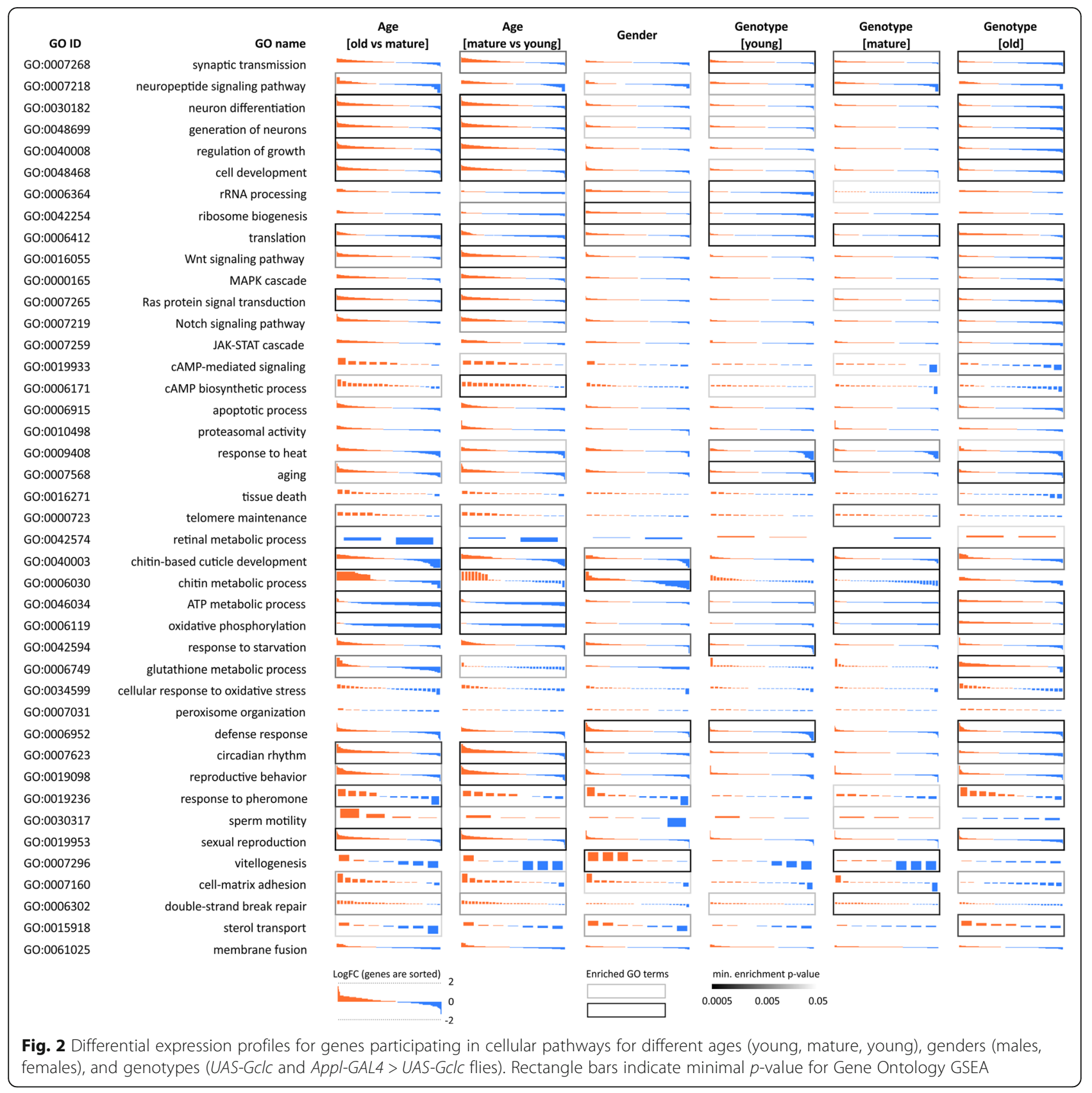

is associated with the low activity level during light time (Fig. 11). At the same time, the decrease of locomotor activity of 4-week-old males with Gclc overexpression is conditioned by the low activity during dark time (Fig. 11). In the connection with alterations in daily activity of males and females, we investigated the influence of Gclc overexpression on the age-dependent dynamics of circadian rhythms.

\section{Circadian activity rhythms}

For the estimation of age-dependent changes of circadian rhythms, we investigated daily changes of locomotor activity of males with Gclc overexpression and without overexpression at the age of 5-13, 30-38, and 50-58 days. At first, the locomotor activity was analyzed during 2 days under conditions of $12 \mathrm{~h}$ light : $12 \mathrm{~h}$ darkness (LD), then 6 days under conditions of $24 \mathrm{~h}$ darkness (DD). It was found that under LD conditions both control and Gclc overexpressing flies at the age of 5 and 30 days demonstrated morning and evening activity peaks before the switching on light, that is defined as zeitgeber time 0 (ZT 0), and switching off light at zeitgeber time 12 (ZT12) compared with 50day-old flies (Fig. 12). At the age of 50 days both 
Table 2 The effect of age on survival of Drosophila after exposure to stresses

\begin{tabular}{|c|c|c|c|c|c|c|c|}
\hline \multirow{3}{*}{$\begin{array}{l}\text { Stress and } \\
\text { genotype of flies }\end{array}$} & \multirow{3}{*}{$\begin{array}{l}\text { Age } \\
\text { (days) }\end{array}$} & \multicolumn{6}{|l|}{ Sex } \\
\hline & & \multicolumn{3}{|l|}{ Male } & \multicolumn{3}{|l|}{ Female } \\
\hline & & $\mathrm{X} \pm \Delta \mathrm{m}$ & $M$ & $90 \%$ & $\mathrm{X} \pm \Delta \mathrm{m}$ & $M$ & $90 \%$ \\
\hline \multirow[t]{9}{*}{ Paraquat UAS-Gclc } & 7 & $76.6 \pm 1.5$ & 84 & 108 & $63.3 \pm 1.7$ & 60 & 96 \\
\hline & 14 & $61.8 \pm 1.6$ & 60 & 84 & $42 \pm 1.4$ & 48 & 72 \\
\hline & 21 & $48 \pm 1.2$ & 48 & 72 & $33 \pm 1.4$ & 36 & 60 \\
\hline & 28 & $36.3 \pm 1.1$ & 36 & 60 & $24.5 \pm 0.9$ & 24 & 48 \\
\hline & 35 & $24.6 \pm 0.9$ & 24 & 48 & $20.5 \pm 0.7$ & 24 & 48 \\
\hline & 42 & $18.2 \pm 0.6$ & 24 & 48 & $17.9 \pm 0.6$ & 24 & 48 \\
\hline & 49 & $17.5 \pm 0.6$ & 24 & 48 & $15 \pm 0.5$ & 24 & 48 \\
\hline & 56 & $15.7 \pm 0.5$ & 24 & 48 & $13.8 \pm 0.4$ & 24 & 48 \\
\hline & 63 & $12.8 \pm 0.2$ & 24 & 48 & $12.3 \pm 0.2$ & 24 & 48 \\
\hline \multirow[t]{10}{*}{ Paraquat Appl-GAL4 > UAS-GClC } & 7 & $80.3 \pm 1.6$ & 84 & 108 & $73.6 \pm 1.8$ & 72 & 108 \\
\hline & 14 & $69.8 \pm 1.6$ & 72 & 96 & $78.2 \pm 1.7$ & 84 & 108 \\
\hline & 21 & $60.4 \pm 1.6$ & 60 & 84 & $53.9 \pm 1.6$ & 60 & 84 \\
\hline & 28 & $52.2 \pm 1.3$ & 48 & 72 & $43.2 \pm 1.2$ & 48 & 72 \\
\hline & 35 & $33 \pm 1.1$ & 36 & 60 & $40.2 \pm 1.1$ & 48 & 72 \\
\hline & 42 & $29.6 \pm 1$ & 36 & 60 & $29.4 \pm 0.9$ & 36 & 60 \\
\hline & 49 & $22.6 \pm 0.8$ & 24 & 48 & $19.2 \pm 0.7$ & 24 & 48 \\
\hline & 56 & $18.8 \pm 0.6$ & 24 & 48 & $19.7 \pm 0.8$ & 24 & 48 \\
\hline & 63 & $16.6 \pm 0.5$ & 24 & 48 & $15 \pm 0.5$ & 24 & 48 \\
\hline & 70 & $14 \pm 0.4$ & 24 & 48 & $13.6 \pm 0.3$ & 24 & 48 \\
\hline \multirow[t]{9}{*}{$\mathrm{CuSO}_{4}$ UAS-GClC } & 7 & $82 \pm 1.4$ & 84 & 108 & $87.3 \pm 1.7$ & 84 & 120 \\
\hline & 14 & $70.2 \pm 1.4$ & 72 & 96 & $76.2 \pm 2$ & 72 & 108 \\
\hline & 21 & $45.4 \pm 1.9$ & 48 & 72 & $60.1 \pm 2.1$ & 60 & 96 \\
\hline & 28 & $46.8 \pm 1.3$ & 48 & 72 & $50.1 \pm 1.9$ & 48 & 84 \\
\hline & 35 & $27 \pm 1.1$ & 24 & 48 & $46.7 \pm 2$ & 48 & 84 \\
\hline & 42 & $31.5 \pm 1$ & 36 & 60 & $43.4 \pm 1.4$ & 48 & 72 \\
\hline & 49 & $21 \pm 0.9$ & 24 & 48 & $29.2 \pm 1.4$ & 24 & 48 \\
\hline & 56 & $20.7 \pm 0.8$ & 24 & 48 & $24.4 \pm 1.1$ & 24 & 48 \\
\hline & 63 & $14.7 \pm 0.4$ & 24 & 48 & $20.6 \pm 0.9$ & 24 & 48 \\
\hline \multirow[t]{10}{*}{$\mathrm{CuSO}_{4} \mathrm{Appl}_{-\mathrm{GAL} 4}>$ UAS-Gclc } & 7 & $96.3 \pm 1.2$ & 96 & 120 & $107 \pm 1.6$ & 108 & 132 \\
\hline & 14 & $78.3 \pm 1.6$ & 84 & 108 & $106 \pm 1.9$ & 108 & 132 \\
\hline & 21 & $52.2 \pm 1.4$ & 48 & 72 & $93.9 \pm 2.1$ & 96 & 120 \\
\hline & 28 & $53.8 \pm 1.4$ & 60 & 84 & $79.1 \pm 2.2$ & 72 & 120 \\
\hline & 35 & $31.7 \pm 1.3$ & 36 & 60 & $60.6 \pm 1.6$ & 60 & 84 \\
\hline & 42 & $32.2 \pm 1$ & 36 & 60 & $57.3 \pm 1.9$ & 60 & 84 \\
\hline & 49 & $23.6 \pm 1$ & 24 & 48 & $52.2 \pm 1.9$ & 60 & 84 \\
\hline & 56 & $25.7 \pm 1$ & 24 & 48 & $37.1 \pm 1.6$ & 36 & 72 \\
\hline & 63 & $16.7 \pm 0.6$ & 24 & 48 & $28.3 \pm 1.4$ & 24 & 48 \\
\hline & 70 & $14.5 \pm 0.4$ & 24 & 48 & $22.2 \pm 1$ & 24 & 48 \\
\hline \multirow[t]{4}{*}{$\mathrm{CdCl}_{2}$ UAS-GClC } & 7 & $67.6 \pm 1.1$ & 72 & 96 & $72.6 \pm 2$ & 72 & 108 \\
\hline & 14 & $55.8 \pm 1.1$ & 60 & 84 & $63.8 \pm 1.9$ & 60 & 96 \\
\hline & 21 & $31.6 \pm 1$ & 36 & 60 & $42.6 \pm 1.8$ & 36 & 72 \\
\hline & 28 & $33 \pm 1$ & 36 & 60 & $43.5 \pm 1.8$ & 36 & 72 \\
\hline
\end{tabular}


Table 2 The effect of age on survival of Drosophila after exposure to stresses (Continued)

\begin{tabular}{|c|c|c|c|c|c|c|c|}
\hline & 35 & $21.9 \pm 0.9$ & 24 & 48 & $38.9 \pm 1.6$ & 36 & 60 \\
\hline & 42 & $25 \pm 0.9$ & 24 & 48 & $32.5 \pm 1.2$ & 36 & 60 \\
\hline & 49 & $16.2 \pm 0.6$ & 24 & 48 & $30.7 \pm 1.5$ & 24 & 60 \\
\hline & 56 & $15.2 \pm 0.5$ & 24 & 48 & $20.4 \pm 0.8$ & 24 & 48 \\
\hline & 63 & $12.8 \pm 0.2$ & 24 & 48 & $12.3 \pm 0.2$ & 24 & 48 \\
\hline \multirow{10}{*}{$\mathrm{CdCl}_{2}$ Appl-GAL4 > UAS-GClC } & 7 & $71.9 \pm 1.7$ & 72 & 96 & $100.1 \pm 1.7$ & 96 & 132 \\
\hline & 14 & $59.9 \pm 1.2$ & 60 & 84 & $91.8 \pm 1.9$ & 96 & 120 \\
\hline & 21 & $39.5 \pm 1.2$ & 48 & 72 & $77 \pm 1.7$ & 84 & 108 \\
\hline & 28 & $41.4 \pm 1.1$ & 48 & 72 & $69 \pm 1.9$ & 72 & 96 \\
\hline & 35 & $27.1 \pm 1$ & 24 & 48 & $51 \pm 1.4$ & 48 & 72 \\
\hline & 42 & $26.3 \pm 0.8$ & 36 & 60 & $44.7 \pm 1.4$ & 48 & 72 \\
\hline & 49 & $19.2 \pm 0.7$ & 24 & 48 & $45.6 \pm 1.3$ & 48 & 72 \\
\hline & 56 & $23.8 \pm 0.7$ & 24 & 48 & $30.7 \pm 1.2$ & 36 & 60 \\
\hline & 63 & $16.8 \pm 0.5$ & 24 & 48 & $22.7 \pm 0.9$ & 24 & 48 \\
\hline & 70 & $15.3 \pm 0.5$ & 24 & 48 & $19.1 \pm 0.8$ & 24 & 48 \\
\hline \multirow[t]{9}{*}{$\mathrm{NaCl}$ UAS-GClC } & 7 & $96.2 \pm 1.6$ & 108 & 132 & 89.84 & 96 & 120 \\
\hline & 14 & $65 \pm 2$ & 60 & 96 & 68.88 & 72 & 96 \\
\hline & 21 & $63.2 \pm 1.7$ & 72 & 96 & 62.72 & 60 & 84 \\
\hline & 28 & $57.6 \pm 1.5$ & 60 & 84 & 58.72 & 60 & 84 \\
\hline & 35 & $34 \pm 1.4$ & 36 & 60 & 46.72 & 48 & 72 \\
\hline & 42 & $30 \pm 1.2$ & 36 & 60 & 32 & 36 & 60 \\
\hline & 49 & $19 \pm 0.7$ & 24 & 48 & 24.8 & 24 & 48 \\
\hline & 56 & $15.4 \pm 0.5$ & 24 & 48 & 16.8 & 24 & 48 \\
\hline & 63 & $12.4 \pm 0.2$ & 24 & 48 & 14.88 & 24 & 48 \\
\hline \multirow[t]{10}{*}{$\mathrm{NaCl}$ Appl-GAL4 > UAS-GClC } & 7 & $85.1 \pm 1.7$ & 84 & 108 & $104.9 \pm 1.8$ & 108 & 132 \\
\hline & 14 & $68 \pm 2$ & 72 & 96 & $85.6 \pm 2.1$ & 96 & 120 \\
\hline & 21 & $61.8 \pm 1.9$ & 60 & 96 & $85.4 \pm 1.8$ & 96 & 120 \\
\hline & 28 & $61 \pm 1.8$ & 60 & 84 & $86.6 \pm 2.2$ & 84 & 120 \\
\hline & 35 & $41.6 \pm 1.7$ & 36 & 72 & $73.8 \pm 1.9$ & 72 & 108 \\
\hline & 42 & $34.4 \pm 1.1$ & 36 & 60 & $56.6 \pm 1.6$ & 60 & 84 \\
\hline & 49 & $20.7 \pm 0.8$ & 24 & 48 & $47 \pm 1.5$ & 48 & 72 \\
\hline & 56 & $17.7 \pm 0.6$ & 24 & 48 & $27 \pm 1.1$ & 24 & 48 \\
\hline & 63 & $15.2 \pm 0.4$ & 24 & 48 & $19 \pm 0.8$ & 24 & 48 \\
\hline & 70 & $14.2 \pm 0.4$ & 24 & 48 & $14.1 \pm 0.4$ & 24 & 48 \\
\hline
\end{tabular}

$\mathrm{X} \pm \Delta \mathrm{m}$ - mean survival (h), $\mathrm{M}$ - median survival time (h), $90 \%$ - the time of $90 \%$ mortality ( $h$ )

short-lived UAS-Gclc flies and long-lived Appl-GAL4 > $U A S-G c l c$ flies had the smoothing of morning and evening activity peaks under LD conditions. The analysis of circadian rhythms of flies that were maintained under DD conditions demonstrated that 50-day-old flies overexpressing Gclc overexpression had more distinct morning and evening activity peaks compared with control flies (Fig. 12).

Additionally, we observed strong overexpression of most genes participating in regulation of circadian rhythms: Per, Tim, Vri, Pdp, $d C L K[15,16]$. In old flies with and without Gclc overexpression, expression of Per, Tim, Vri, and $d C L K$ was slightly down-regulated (Additional file 1). Obtained data demonstrated that Gclc overexpression led to slowing of age-dependent disruption of circadian activity.

\section{Discussion}

We have found that the lifespan expending effect of overexpression of the Gclc gene in the nervous system protects from the age-dependent disruption of circadian rhythmicity and locomotor activity. This effect can be 

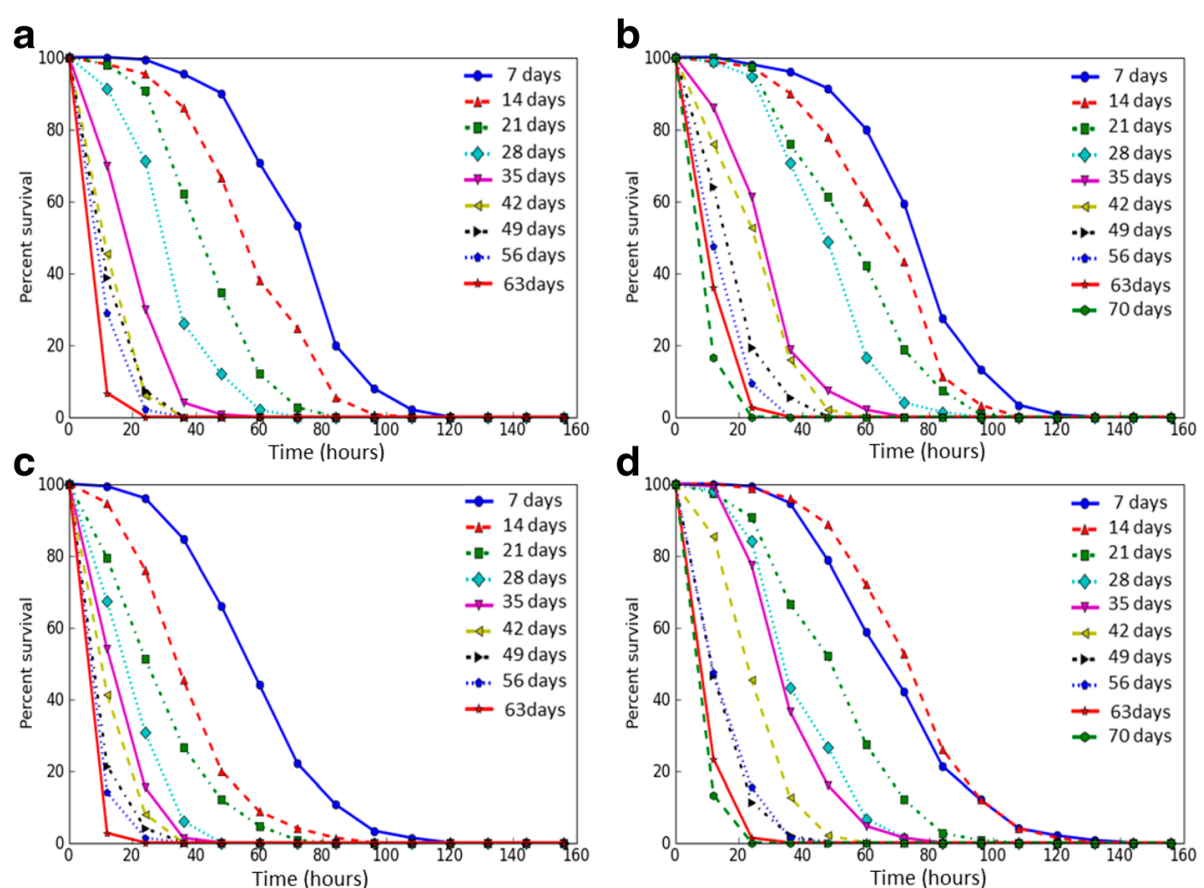

Fig. 3 The survival curves of Drosophila melanogaster in different ages upon exposure to paraquat. a - Appl-GAL4 > UAS-Gclc males; b - UAS-GClc males; c - Appl-GAL4 > UAS-Gclc females; d - UAS-Gclc females
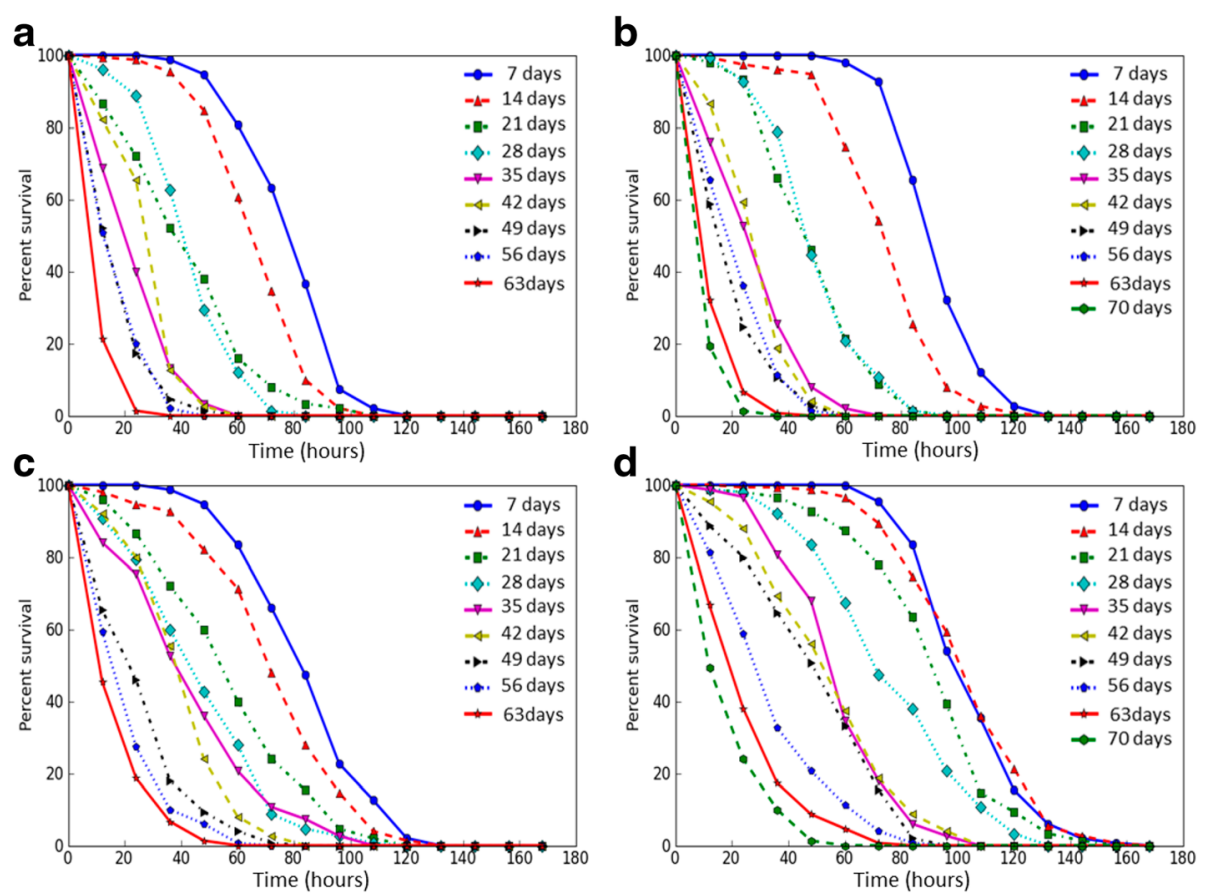

Fig. 4 The survival curves of Drosophila melanogaster in different ages upon exposure to CuSO 4 . a - Appl-GAL4 > UAS-Gclc males; $\mathbf{b}$ - UAS-Gclc males; c - Appl-GAL4 > UAS-Gclc females; d - UAS-Gck females 

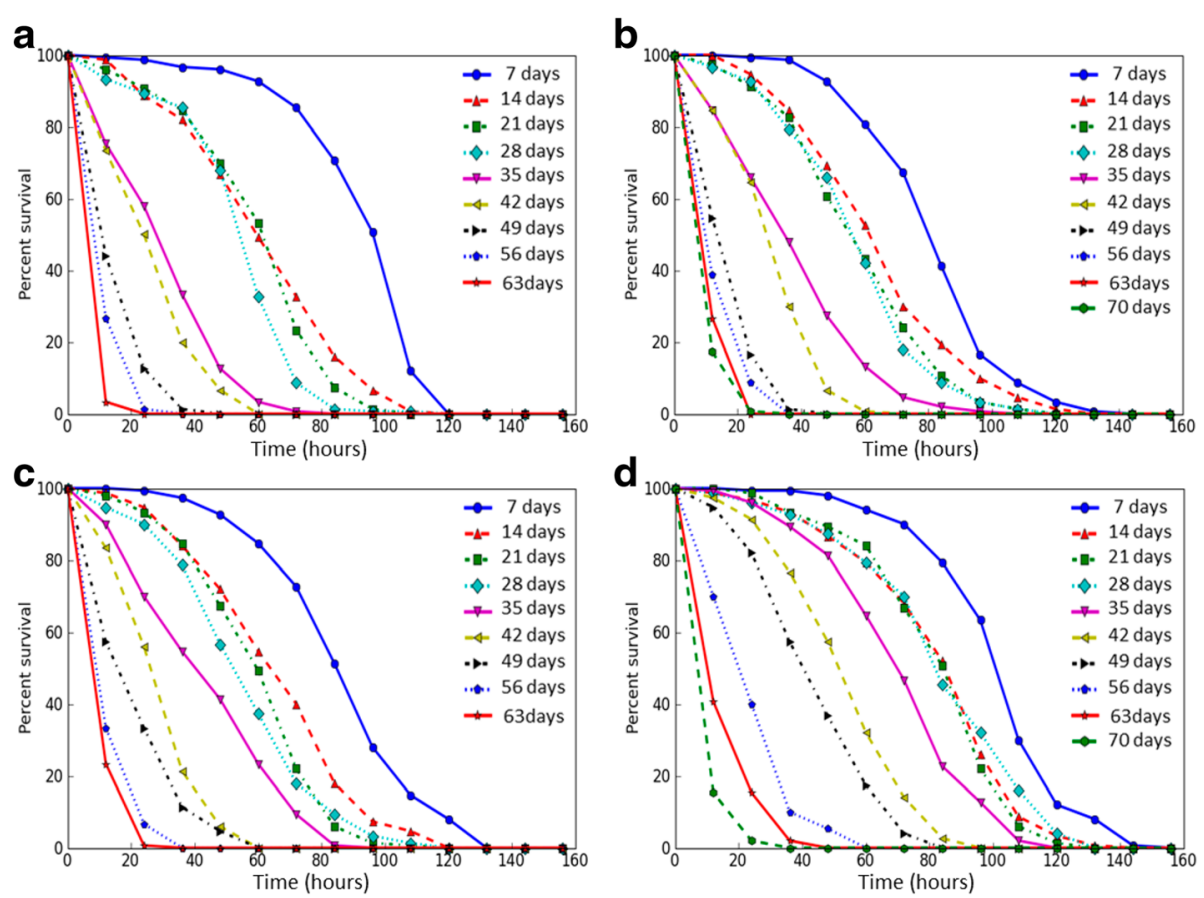

Fig. 5 The survival curves of Drosophila melanogaster in different ages upon exposure to $\mathrm{CdCl}_{2}$. $\mathbf{a}$ - Appl-GAL4 > UAS-Gclc males; b - UAS-Gclc males; c - Appl-GAL4 > UAS-Gclc females; d - UAS-Gclc females
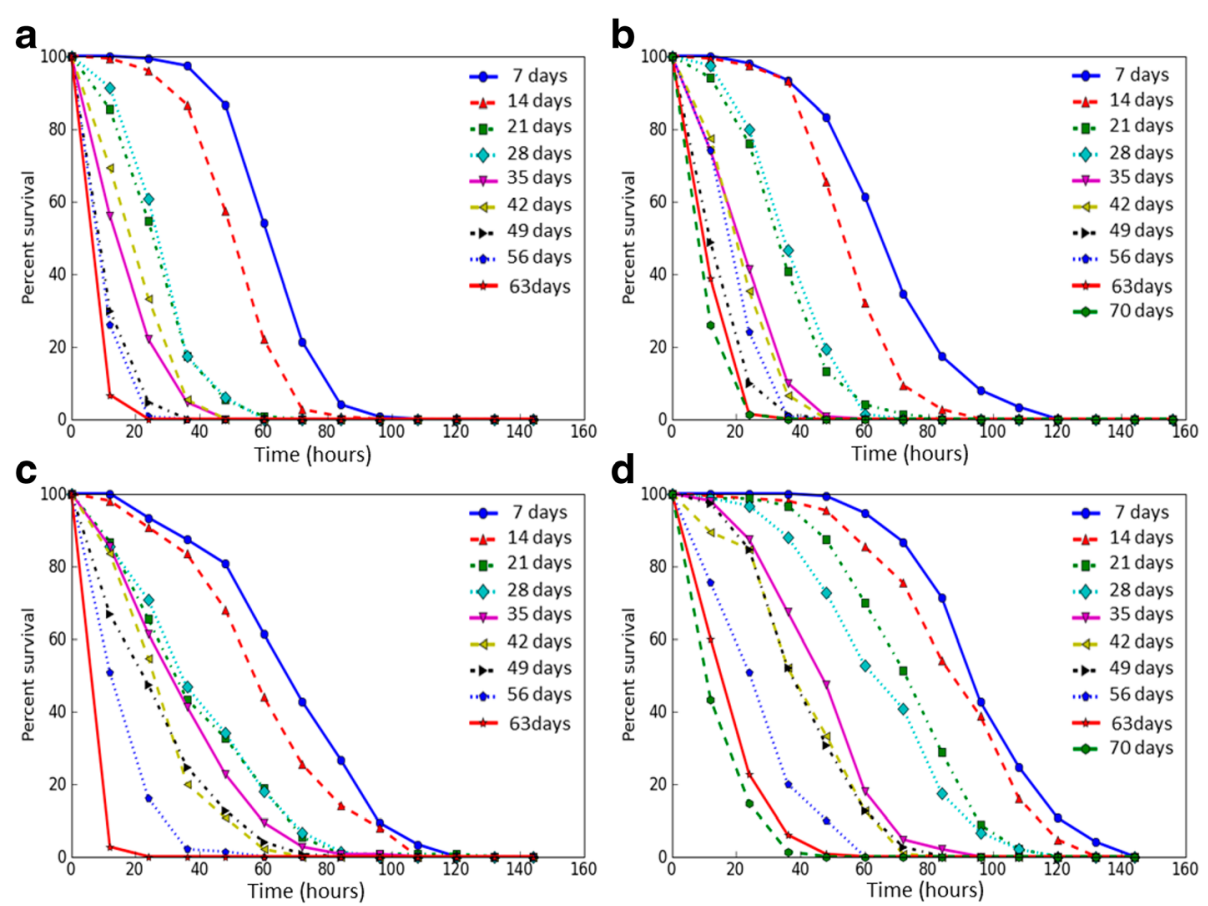

Fig. 6 The survival curves of Drosophila melanogaster in different ages upon exposure to NaCl. a - Appl-GAL4 > UAS-Gclc males; b - UAS-Gclc males; c - Appl-GAL4 > UAS-Gclc females; d - UAS-Gclc females 
Table 3 Cox regression calculation results for Drosophila after exposure to different stress factors

\begin{tabular}{|c|c|c|c|c|c|c|}
\hline \multirow[t]{2}{*}{ Genotype } & \multirow[t]{2}{*}{ Factor } & \multirow[t]{2}{*}{ Parameter } & \multicolumn{4}{|l|}{ Stresses } \\
\hline & & & Paraquat & $\mathrm{CuSO}_{4}$ & $\mathrm{CdCl}_{2}$ & $\mathrm{NaCl}$ \\
\hline \multirow[t]{2}{*}{ Appl-GAL4 > UAS-Gclc } & \multirow[t]{2}{*}{ Sex } & $\mathrm{HR}$ & 1.026 & 0.5152 & 0.4533 & 0.4533 \\
\hline & & $95 \% \mathrm{Cl}$ & $0.9546-1.102$ & $0.4783-0.555$ & $0.4201-0.4891$ & $0.4201-0.4891$ \\
\hline \multirow[t]{8}{*}{ UAS-GCKC } & \multirow[t]{2}{*}{ Age } & $H R$ & 1.078 & 1.0681 & 1.0654 & 1.0824 \\
\hline & & $95 \% \mathrm{Cl}$ & $1.0757-1.0802$ & $1.0661-1.0701$ & $1.0634-1.0674$ & $1.0800-1.0848$ \\
\hline & \multirow[t]{2}{*}{ Sex } & $H R$ & 1.5808 & 0.5105 & 0.4301 & 0.769 \\
\hline & & $95 \% \mathrm{Cl}$ & $1.4650-1.7057$ & $0.4724-0.5516$ & $0.3975-0.4655$ & $0.7128-0.8297$ \\
\hline & \multirow{2}{*}{$\begin{array}{l}\text { Sex_male: } \\
\text { Appl-GAL4 > UAS-GClC }\end{array}$} & $H R$ & 0.5333 & 0.710595 & 0.5943 & 0.7561 \\
\hline & & $95 \% \mathrm{Cl}$ & $0.4947-0.5749$ & $0.6597-0.7651$ & $0.5513-0.6407$ & $0.7015-0.8150$ \\
\hline & \multirow{2}{*}{$\begin{array}{l}\text { Sex_female: } \\
\text { Appl-GAL4 > UAS-Gclc }\end{array}$} & $H R$ & 0.3558 & 0.3914 & 0.4064 & 0.3727 \\
\hline & & $95 \% \mathrm{Cl}$ & $0.3296-0.3841$ & $0.3625-0.4226$ & $0.3769-0.4383$ & $0.3451-0.4025$ \\
\hline
\end{tabular}

The first column shows the genotype compared flies in the analysis of the impact of these factors in the second column. HR - hazard ratio (the predicted risk of change when the value of the independent variable on the unit); $95 \% \mathrm{Cl}$ - a $95 \%$ confidence interval; Sex - a series of data showing the hazard regression for the flies with a certain genotype under the influence of each of the stress when factor is the individual's sex; Age - a series of data showing the hazard regression for the flies with a certain genotype under the influence of each of the stress when factor is the individual's age; Sex_male: Appl-GAL4 > UAS-Gclc a series of data showing the hazard ratio for flies with UAS-Gclc and Appl-GAL4 > UAS-Gclc genotypes when exposed to each of stresses, with the assessment of the impact of interacting factors (male sex, and genotype Appl-GAL4 > UAS-Gclc); Sex_female: Appl-GAL4 > UAS-Gclc a series of data showing the hazard ratio for flies with UAS-Gclc and Appl-GAL4 > UAS-Gclc genotypes when exposed to each of stresses, with the assessment of the impact of interacting factors (female sex, and genotype Appl-GAL4 > UAS-Gclc)

mediated by the activation of genes participating in regulation of circadian rhythms. Despite the lack of the effect of Gclc overexpression to fertility, we found increased activity of many genes participating in the reproductive function.

Table 4 Dependence of the average survival age of Drosophila after exposure to stresses

\begin{tabular}{|c|c|c|c|c|c|}
\hline \multicolumn{3}{|c|}{ Variant of the experiment } & \multirow[t]{2}{*}{$N$} & \multirow[t]{2}{*}{ Pearson $r$} & \multirow[t]{2}{*}{$p$} \\
\hline Stress & Sex & Genotype & & & \\
\hline Paraquat & Male & UAS-GClC & 9 & -0.944 & $<0.001$ \\
\hline araquat & Male & Appl-GAL4 > UAS-Gclc & 10 & -0.968 & $<0.001$ \\
\hline $\mathrm{uSO}_{4}$ & Male & UAS-GClC & 9 & -0.941 & $<0.001$ \\
\hline $\mathrm{CuSO}_{4}$ & Male & Appl-GAL4 > UAS-Gclc & 10 & -0.934 & $<0.001$ \\
\hline $\mathrm{CdCl}_{2}$ & Male & UAS-GClC & 9 & -0.914 & $<0.001$ \\
\hline $\mathrm{CdCl}_{2}$ & Male & Appl-GAL4 > UAS-Gclc & 10 & -0.922 & $<0.001$ \\
\hline $\mathrm{NaCl}$ & Male & UAS-GClC & 9 & -0.964 & $<0.001$ \\
\hline $\mathrm{NaCl}$ & Male & Appl-GAL4 > UAS-Gclc & 10 & -0.974 & $<0.001$ \\
\hline araquat & Female & UAS-GClC & 9 & -0.902 & $<0.001$ \\
\hline araquat & Female & Appl- & 10 & -0.958 & $<0.001$ \\
\hline $\mathrm{CuSO}_{4}$ & Female & UAS-GCKC & 9 & -0.981 & $<0.001$ \\
\hline $\mathrm{CuSO}_{4}$ & Female & Appl-GAL4 > UAS-Gclc & 10 & -0.990 & $<0.001$ \\
\hline $\mathrm{CdCl}_{2}$ & Female & UAS-GCKC & 9 & -0.967 & $<0.001$ \\
\hline $\mathrm{CdCl}_{2}$ & Female & Appl-GAL4 > UAS-GClC & 10 & -0.986 & $<0.001$ \\
\hline $\mathrm{NaCl}$ & Female & UAS-GCKC & 9 & -0.986 & $<0.001$ \\
\hline $\mathrm{laCl}$ & Female & Appl-GAL4 > UAS-Gclc & 10 & -0.978 & $<0.001$ \\
\hline
\end{tabular}

$N$ - the number of variables (median survival of flies at a certain age), Pearson $r$ - the Pearson correlation coefficient, $p$ - the level of significance of the correlation coefficient
Overall, transcriptomic data demonstrated the activation of apoptosis pathways, Wnt, mTOR/PI3K, FOXO pathways, circadian rhythm genes, enhanced cAMP signaling, overexpression of Myc and Notch, as well as the decrease of mitochondrial function, oxidative phosphorylation, ribosome biogenesis, and translation as one of the hallmarks of aging. Previously, oxidative phosphorylation and related activities were found as only processes that are down-regulated in whole insect body according to the results of three previous transcriptomic studies of aging Drosophila [17-19]. Impairment of oxidative phosphorylation during aging is well known for other model organisms [20]. On the other hand, the stress response, including defense response and amino acid and purine metabolism, was found to be up-regulated during aging [21]. This is not supported with our data.

However, there is no great consensus between the different studies concerning other biological processes. Our results particularly contradict the data of Fabrice Girardot et al. [17]. Using microarray analysis, the authors have found that neuronal activity, especially response to light, is repressed during aging (3 days vs. 40 days old Drosophila melanogaster). In contrast, we found the predominant up-regulation of genes in the categories related to the neuronal function, excluding the neuropeptide signaling pathway, which was featured with the predominant down-regulation of expression. However, genes responsible for retinal metabolic processes were also found significantly repressed during aging. We confirmed that guanylyl cyclase alpha-subunit 99B (Gyca99B), which was previously found by Girardot et al. [17] as the most strongly associated with aging, 

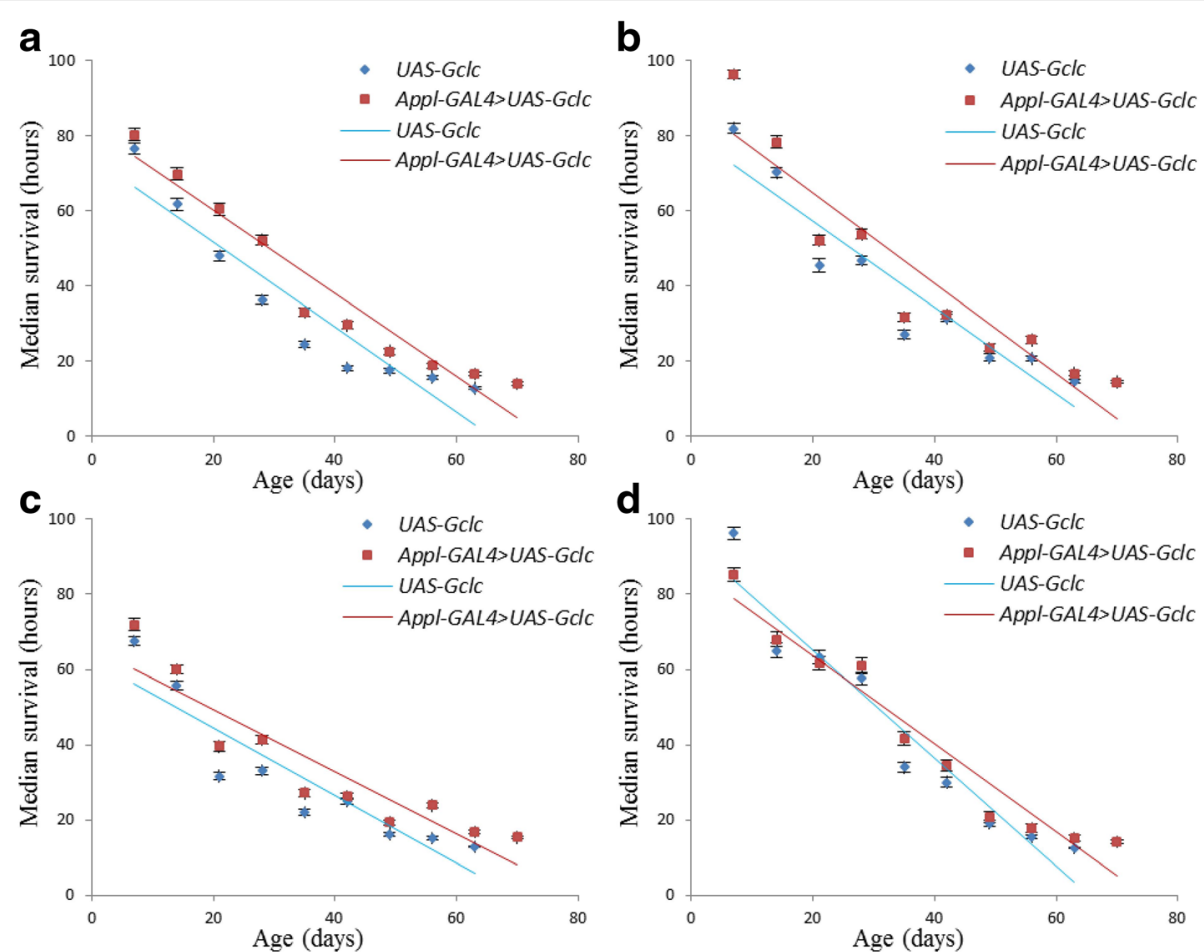

Fig. 7 The dependence of survival on the age of Appl-GAL4 > UAS-GClc and UAS-Gclc males. a - paraquat; b - CuSO $;$; $-\mathrm{CdCl}_{2} ; \mathbf{d}-\mathrm{NaCl}$. Color lines - linear regression
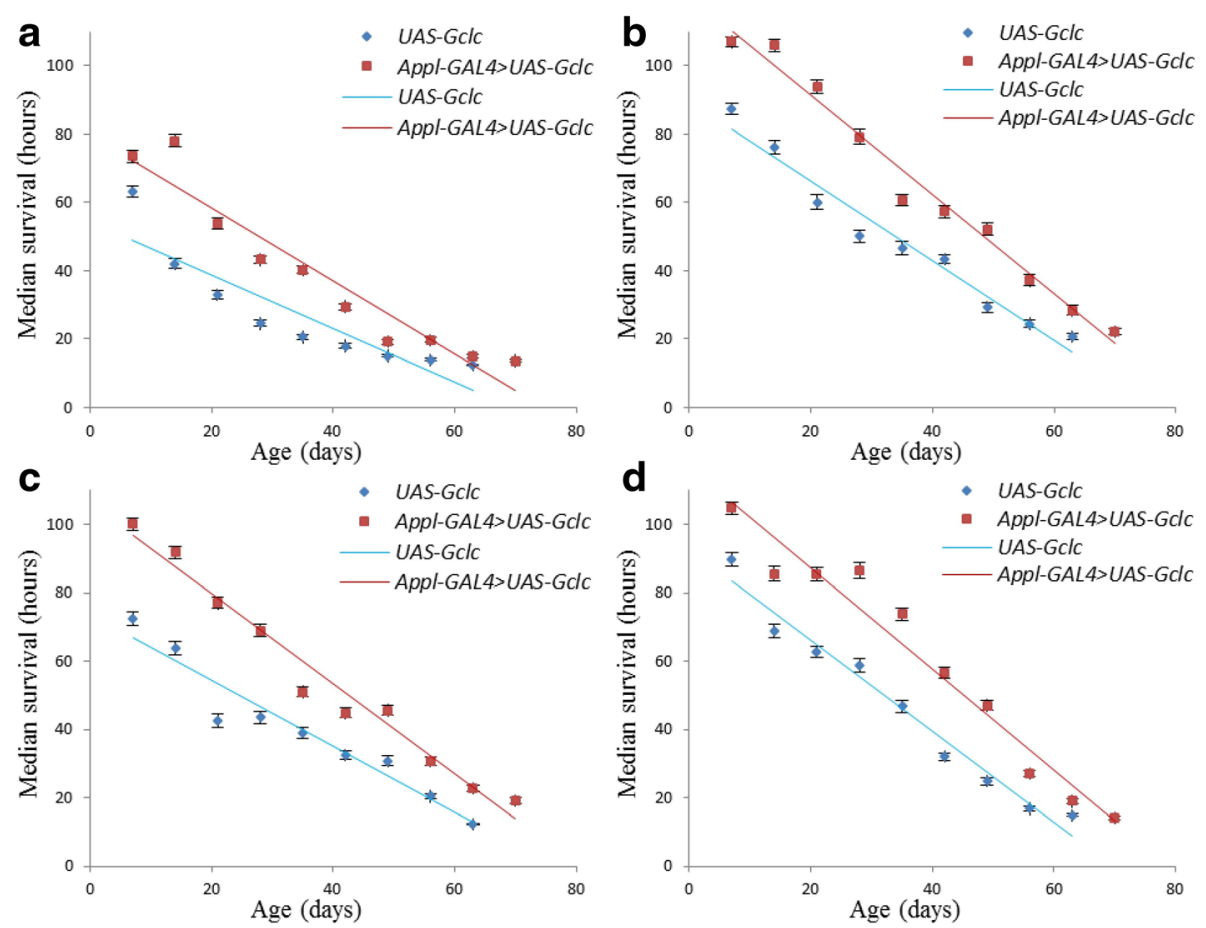

Fig. 8 The dependence of survival on the age of Appl-GAL4 > UAS-Gclc and UAS-Gclc females. a - paraquat; b - CuSO $;$; $-\mathrm{CdCl}_{2} ; \mathbf{d}-\mathrm{NaCl}_{\text {. Color }}$ lines - linear regression 
Table 5 The significance of differences of correlation coefficients for Drosophila after exposure to stresses

\begin{tabular}{lll}
\hline Stress & Sex & $p$ \\
\hline Paraquat & Male & 0.30 \\
$\mathrm{CuSO}_{4}$ & Male & 0.44 \\
$\mathrm{CdCl}_{2}$ & Male & 0.46 \\
$\mathrm{NaCl}$ & Male & 0.40 \\
Paraquat & Female & 0.20 \\
$\mathrm{CuSO}_{4}$ & Female & 0.27 \\
$\mathrm{CdCl}_{2}$ & Female & 0.17 \\
$\mathrm{NaCl}$ & Female & 0.27 \\
\hline
\end{tabular}

$p$ - the significance level of the difference coefficients

demonstrates the perfect anti-correlation with aging according to our data (Spearman's rank correlation coefficient $r=-0.88, p<10^{-5}$ ). In 2011, Pernille Sarup et al. found that longevity-selected lines of Drosophila melanogaster retain the young gene expression profile including differential expression of starvation response genes, down-regulation of genes participating in immune response and possibly, chronic inflammation [22].

Despite the fact that the changes of transcriptomic profiles introduced by activated Gclc are not so pronounced as the age- or gender-associated alterations, Gclc-associated changes are related to key processes that are involved in aging. Many genes that comprise 'tissue death' and 'aging' GO terms are up-regulated during aging of Drosophila melanogaster, but Gclc enhanced activity induced suppression of many genes related to these categories in old insects with Gclc overexpression (Fig. 2). Gclc inversed the aging-associated repression of cell respiration translation: it induced the upregulation of oxidative phosphorylation genes and ribosomal genes suggesting the overall increase of protein synthesis. The similar observation is true for cAMP pathways: overactivated Gclc downregulated the most of genes involved in cAMP signaling, activation of which is one of the aging hallmarks [23-25].

The analysis of KEGG pathways demonstrated Gclc-induced alterations in key cell signaling pathways that are involved in aging of a cell and organism. This effect was primarily observed for old individuals, but wasn't detected for middle-aged and young imagoes. In aged flies with Gclc overexpression compared with control insects, we observed: decrease of many genes comprising MAPK pathway associated with aging and stress [26, 27], agingassociated mTOR/PI3K pathway [28], and FOXO. However, the latter one is thought to be a contributor to longevity, stress resistance and tumor suppression [29].

The expression of eight genes, SMC2 (Structural maintenance of chromosomes 2), w (white), CG4293, Gclc, Cyp4p2 (cytochrome P450 4p2), Ipk1 (Inositol-pentakisphosphate 2-kinase), CG8157, CR45457 demonstrated strikingly strong association with Gclc overexpression in all of the groups: young/mature/old or males/females. We observed 1.5-4-fold decrease of various Turandot family members (TotC, TotM, TotX, TotA) in Drosophila melanogaster with Gclc overactivation of all ages (young, mature and old). Members of this family are induced in the response to various stresses including starvation, toxic substances, irradiation, infectious agents $[6,30]$. Most likely, down-regulation of the Turandot family is a result of the overall reduction in stress load (including oxidative stress).

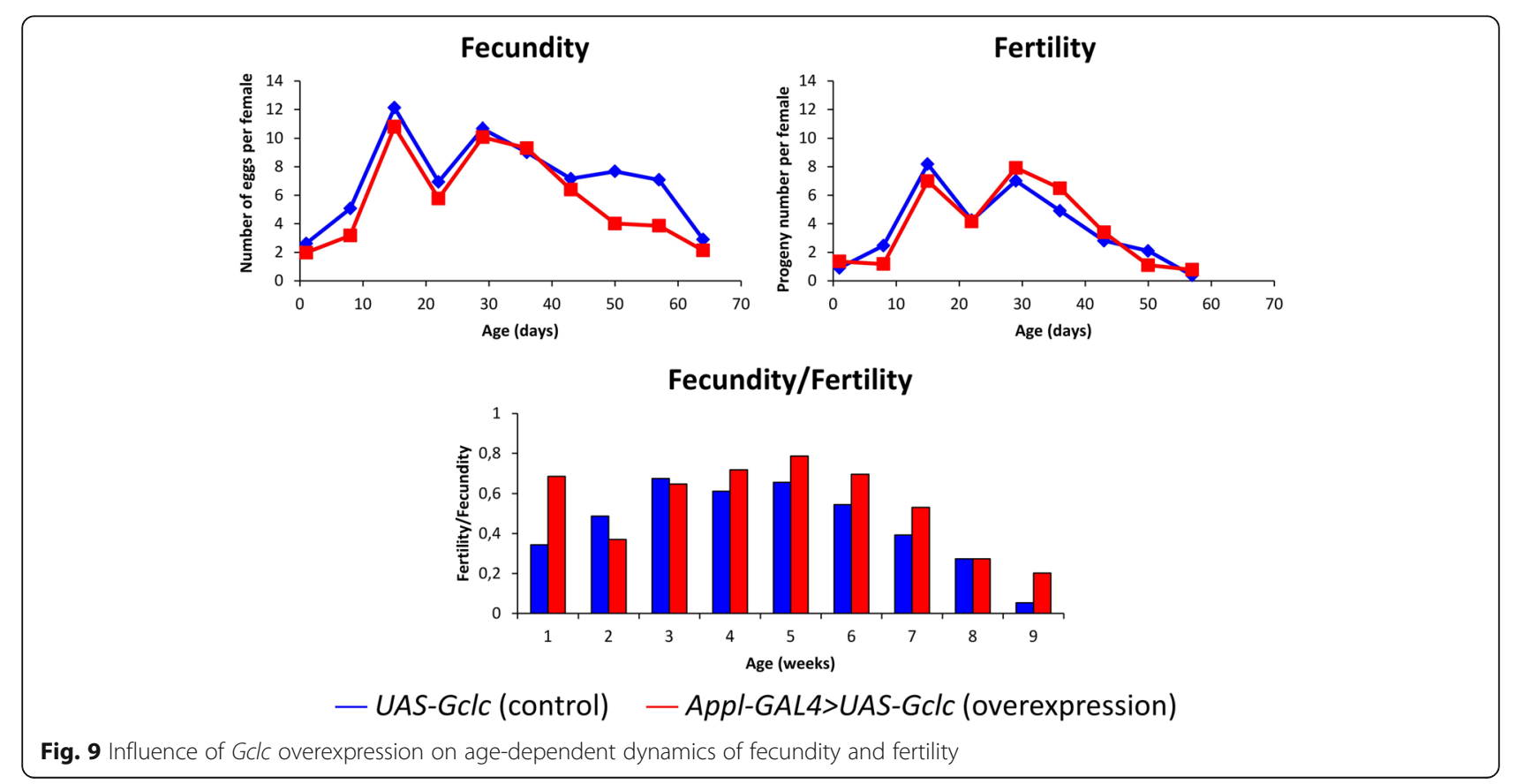




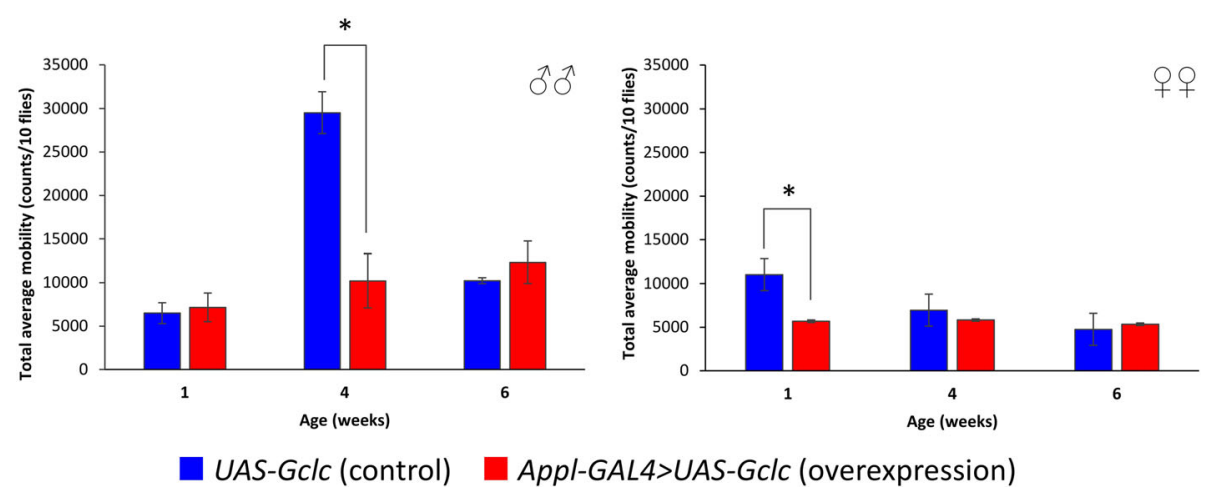

Fig. 10 Influence of Gclc overexpression on age-dependent dynamics of total daily locomotor activity. ${ }^{*} p<0.05, t-S t u d e n t$ test

This hypothesis is indirectly supported by the predominant decrease of many genes of category 'defense response' as well as heat shock proteins responsible for protein refolding ('response to heat' terms). The expression of Tot genes is regulated by JAK-STAT and MAPK pathways [31]. The elements of these two pathways were significantly down-regulated in flies with Gclc overexpression, according to the KEGG pathway analysis (Additional file 1).

Analysis of the influence of age on the survival of Drosophila melanogaster with constitutive overexpression of the Gclc gene and control flies in oxidative, proteotoxic and osmotic stress conditions showed that both genotypes characterized by the negative correlation between age and stress tolerance. The gradual deterioration of the ability to respond to stress with age may be a general aging mechanism for different organisms [32-34]. For Drosophila melanogaster and a number of other species it has been shown that the increase in lifespan is accompanied by the impairment of the resistance to various stress factors [35-37]. On the other hand, accelerated aging is often accompanied by a decrease in stress

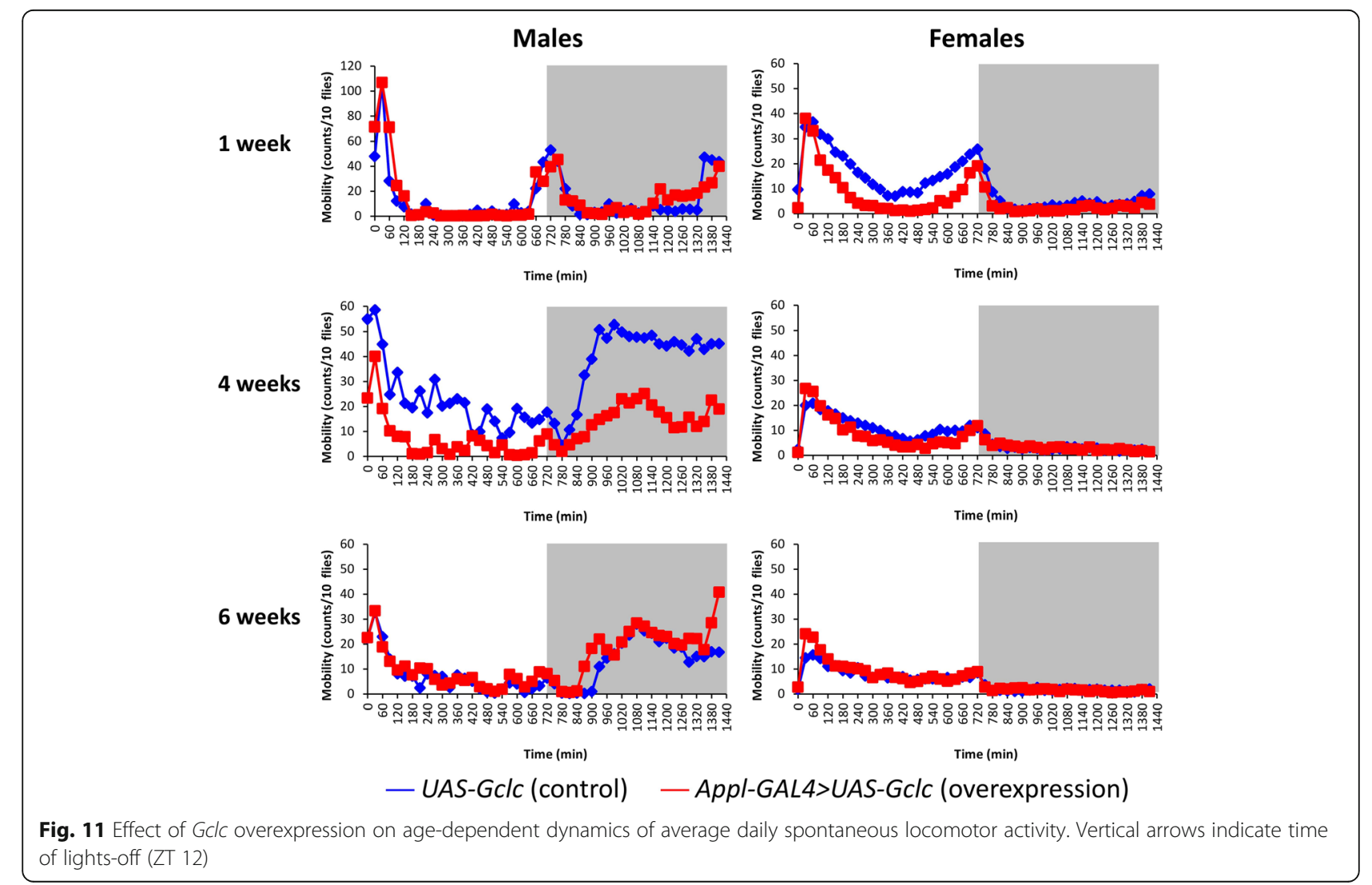



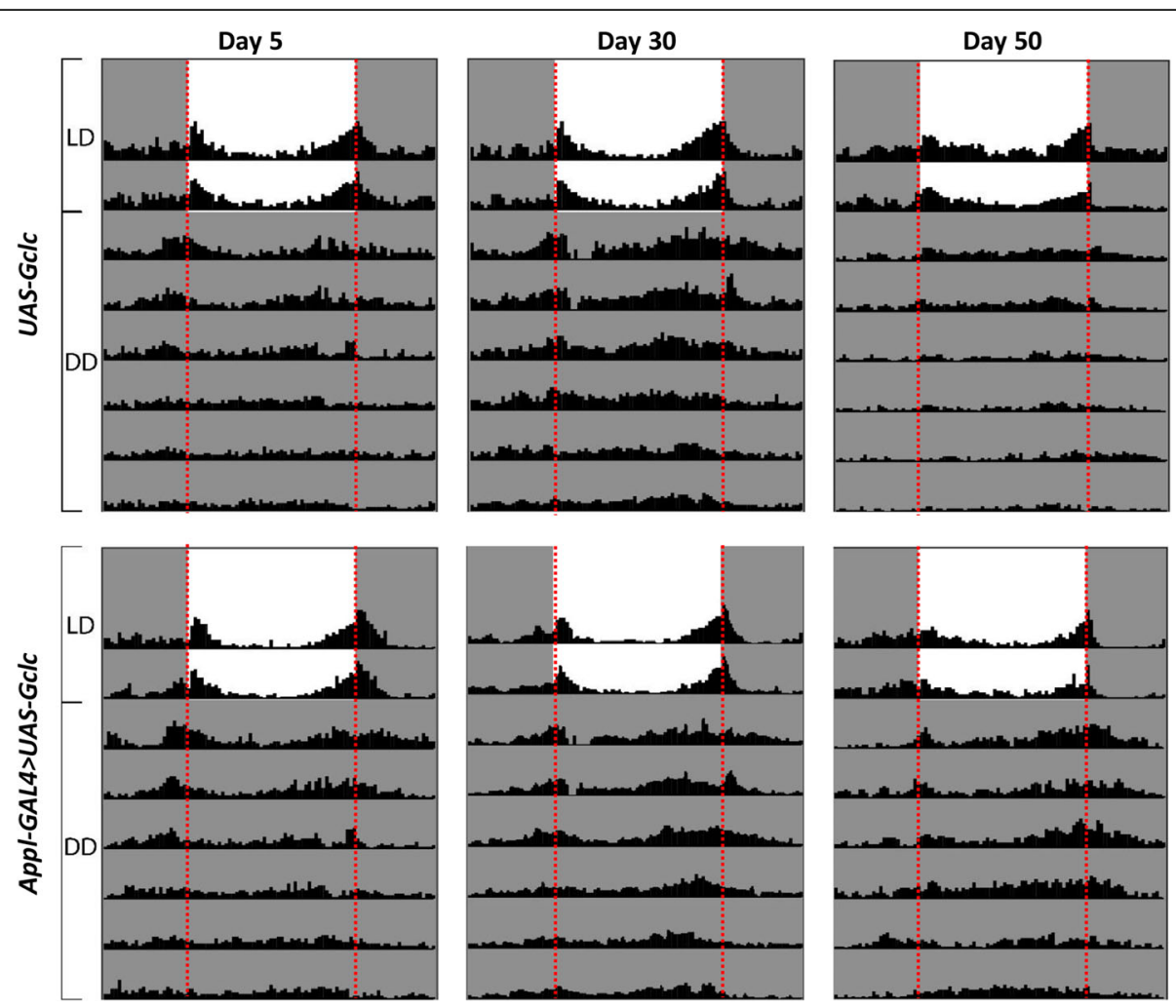

Fig. 12 Influence of Gclc overexpression on age-dependent disruption of locomotor activity rhythms. Locomotor activity profiles of representative 5-, 30-, and 50-day-old males of the indicated genotypes. Flies of each age were monitored in LD (12:12) for 2-d, followed by 6-d in DD at $25^{\circ} \mathrm{C}$. Shaded areas represent periods of darkness. Vertical red dotted lines indicate time of lights-on (ZT/CT 0) and lights-off (ZT/CT 12)

tolerance [38]. Additionally, our study found no significant difference in the dynamics of stress tolerance at different ages throughout the entire range of the studied stresses, as well as in different sexes. This supports the idea that there is an infringement of physiological functions and homeostasis associated with aging, such changes are common to the different sexes and affect a wide range of stress response mechanisms [39].

Overall, the survival in the conditions of oxidative, proteotoxic and osmotic stresses of flies overexpressing the Gclc gene is significantly higher compared with control flies. These stresses are directly or indirectly lead to increased oxidative stress in the body. Therefore elevated levels of GSH has a positive effect on the survival of flies $[40,41]$. Aging reduces the content of GSH in cells, as well as disturbs maintaining a certain level of GSH at oxidative stress conditions [42]. One of mechanisms of the occurrence of these effects may be the violation of GSH synthesis mechanisms and its affinity for the substrate [43]. Thus, Gclc overexpression increases the organism ability to resist stress at all ages, but it is not able to slow down the dynamics of the reduction of stress resistance.

\section{Conclusion}

Our study revealed that Gclc overexpression induces transcriptional changes associated with lifespan extension and uncovered pathways that may be associated with the age-dependent decline of biological functions. The Gclc level demonstrated associations with expression of genes involved in a variety of cellular processed including Jak-STAT, MAPK, FOXO, Notch, mTOR, TGF-beta signaling pathways, translation, protein processing in endoplasmic reticulum, proteasomal degradation, glycolysis, oxidative phosphorylation, apoptosis, regulation of circadian rhythms, differentiation of neurons, synaptic plasticity and transmission.

\section{Additional file}

Additional file 1: Upregulated and downregulated pathways changing activity during aging of flies with overexpression of the Gclc gene and without overexpression based on KEGG data. This figure shows both genes with statistically significant differential expression $(p<0.05)$ and genes that only demonstrate up/down-regulation trends (statistically insignificant; almost all of them have low absolute LogFC values and are marked with light shades). (PDF $1137 \mathrm{~kb}$ ) 


\section{Abbreviations}

Gclc: Glutamate-cysteine ligase catalytic subunit

\section{Acknowledgments}

Authors thank Institute of Biology and Authors thank Institute of Biology Komi SC UrB RAS and Moscow Institute of Physics and Technology for scientific advice and Drosophila strains. Part of this work was performed using EIMB RAS "Genome" center equipment (http://www.eimb.ru/ RUSSIAN_NEW/INSTITUTE/ccu_genome_c.php).

\section{Declarations}

This article has been published as part of BMC Genetics Vol 17 Suppl 14, 2016 : Selected articles from BGRSISB-2016: genomics. The full contents of the supplement are available online at http://bmcgenomics.biomedcentral.com/ articles/supplements/volume-17-supplement-14.

\section{Funding}

This work and publication costs were funded by the Russian Science Foundation grant N 14-50-00060.

\section{Availability of data and material}

The sequencing data are available through the NCBI Sequence Read Archive (project ID PRJNA343315). Upregulated and downregulated pathways based on KEGG data are provided in the Additional file 1.

\section{Authors' contributions}

AM, MS, EP, GK, AK wrote the manuscript text. MS, EP, SZ, AS, AS, ZG carried out the experiments. AM, GK, SZ, AK carried out the bioinformatic analysis. AM and AK supervised the bioinformatic research and text of the manuscript. All authors read and approved the final manuscript.

\section{Competing interests}

The authors declare that they have no competing interests.

\section{Consent for publication}

Not applicable.

\section{Ethics approval and consent to participate}

Not applicable.

\section{Author details}

'Engelhardt Institute of Molecular Biology, Russian Academy of Sciences, Moscow, Russia. ${ }^{2}$ Institute of Biology of Komi Science Center of Ural Branch of RAS, Syktyvkar, Russia. ${ }^{3}$ Moscow Institute of Physics and Technology, Dolgoprudny, Russia. ${ }^{4}$ Syktyvkar State University, Syktyvkar, Russia.

\section{Published: 28 December 2016}

\section{References}

1. Orr WC, Radyuk SN, Prabhudesai L, Toroser D, Benes JJ, Luchak JM, Mockett RJ, Rebrin I, Hubbard JG, Sohal RS. Overexpression of glutamate-cysteine ligase extends life span in Drosophila melanogaster. J Biol Chem. 2005;280: 37331-8.

2. Mårtensson J, Jain A, Stole E, Frayer W, Auld P, Meister A. Inhibition of glutathione synthesis in the newborn rat: a model for endogenously produced oxidative stress. Proc Natl Acad Sci U S A. 1991;88:9360-4.

3. Moore WR, Anderson ME, Meister A, Murata K, Kimura A. Increased capacity for glutathione synthesis enhances resistance to radiation in Escherichia coli: a possible model for mammalian cell protection. Proc Natl Acad Sci U S A. 1989;86:1461-4

4. Tower J. Transgenic methods for increasing Drosophila life span. Mech Ageing Dev. 2000;118:1-14

5. Wang C, Li Q, Redden DT, Weindruch R, Allison DB. Statistical methods for testing effects on "maximum lifespan". Mech Ageing Dev. 2004;125:629-32.

6. Moskalev A, Zhikrivetskaya S, Krasnov G, Shaposhnikov M, Proshkina E, Borisoglebsky D, Danilov A, Peregudova D, Sharapova I, Dobrovolskaya E, Solovev I, Zemskaya N, Shilova L, Snezhkina A, Kudryavtseva A. A comparison of the transcriptome of Drosophila melanogaster in response to entomopathogenic fungus, ionizing radiation, starvation and cold shock. BMC Genomics. 2015;16 Suppl 13:S8.
7. Moskalev A, Shaposhnikov M, Snezhkina A, Kogan V, Plyusnina E, Peregudova D, Melnikova N, Uroshlev L, Mylnikov S, Dmitriev A, Plusnin S, Fedichev $P$, Kudryavtseva A. Mining gene expression data for pollutants (dioxin, toluene, formaldehyde) and low dose of gamma-irradiation. PLoS One. 2014;9, e86051

8. Krasnov GS, Dmitriev AA, Kudryavtseva AV, Shargunov AV, Karpov DS, Uroshlev LA, Melnikova NV, Blinov VM, Poverennaya EV, Archakov Al, Lisitsa AV, Ponomarenko EA. PPLine: An Automated Pipeline for SNP, SAP, and Splice Variant Detection in the Context of Proteogenomics. J Proteome Res. 2015;14:3729-37.

9. Robinson MD, McCarthy DJ, Smyth GK. edgeR: a Bioconductor package for differential expression analysis of digital gene expression data. Bioinformatics. 2010;26:139-40.

10. $Y u$ G, Wang LG, Han Y, He QY. clusterProfiler: an R package for comparing biological themes among gene clusters. OMICS. 2012;16:284-7.

11. Luo W, Brouwer C. Pathview: an R/Bioconductor package for pathway-based data integration and visualization. Bioinformatics. 2013:29:1830-1.

12. Yang J-S, Nam H-J, Seo M, Han SK, Choi Y, Nam HG, Lee S-J, Kim S. OASIS: Online Application for the Survival Analysis of Lifespan Assays Performed in Aging Research. PLoS One. 2011;6, e23525.

13. Schmid B, Helfrich-Forster C, Yoshii T. A new ImageJ plug-in "ActogramJ" for chronobiological analyses. J Biol Rhythms. 2011;26:464-7.

14. Schneider CA, Rasband WS, Eliceiri KW. NIH Image to ImageJ: 25 years of image analysis. Nat Methods. 2012;9:671-5.

15. Tataroglu O, Emery P. The molecular ticks of the Drosophila circadian clock. Curr Opin Insect Sci. 2015;7:51-7.

16. Nikhil KL, Abhilash L, Sharma VK. Molecular Correlates of Circadian Clocks in Fruit Fly Drosophila melanogaster Populations Exhibiting early and late Emergence Chronotypes. J Biol Rhythms. 2016;31:125-41.

17. Girardot F, Lasbleiz C, Monnier V, Tricoire H. Specific age-related signatures in Drosophila body parts transcriptome. BMC Genomics. 2006;7:69.

18. Broughton SJ, Piper MD, Ikeya T, Bass TM, Jacobson J, Driege $Y$, Martinez $P$, Hafen E, Withers DJ, Leevers SJ, Partridge L. Longer lifespan, altered metabolism, and stress resistance in Drosophila from ablation of cells making insulin-like ligands. Proc Natl Acad Sci U S A. 2005;102:3105-10.

19. Clancy DJ, Gems D, Harshman LG, Oldham S, Stocker H, Hafen E, Leevers SJ, Partridge L. Extension of life-span by loss of CHICO, a Drosophila insulin receptor substrate protein. Science. 2001:292:104-6.

20. Lesnefsky EJ, Hoppel CL. Oxidative phosphorylation and aging. Ageing Res Rev. 2006:5:402-33.

21. Landis GN, Abdueva D, Skvortsov D, Yang J, Rabin BE, Carrick J, Tavare S, Tower J. Similar gene expression patterns characterize aging and oxidative stress in Drosophila melanogaster. Proc Natl Acad Sci U S A. 2004;101:7663-8.

22. Sarup $P$, Sorensen $P$, Loeschcke $V$. Flies selected for longevity retain a young gene expression profile. Age (Dordr). 2011;33:69-80.

23. Park SJ, Ahmad F, Philp A, Baar K, Williams T, Luo H, Ke H, Rehmann H, Taussig R, Brown AL, Kim MK, Beaven MA, Burgin AB, Manganiello V, Chung $J$. Resveratrol ameliorates aging-related metabolic phenotypes by inhibiting CAMP phosphodiesterases. Cell. 2012;148:421-33.

24. Coelho Horta B, Steinberg Perilo C, Caldeira Costa D, Nogueira-Machado JA, Martins Chaves M. Aging: functional metabolic balance among CAMP, cGMP and reactive oxygen intermediate generation by human granulocytes. Gerontology. 2005;51:363-8.

25. Liu AY, Chang ZF, Chen KY. Increased level of CAMP-dependent protein kinase in aging human lung fibroblasts. J Cell Physiol. 1986;128:149-54.

26. Papaconstantinou J, Wang CZ, Zhang M, Yang S, Deford J, Bulavin DV, Ansari $\mathrm{NH}$. Attenuation of p38alpha MAPK stress response signaling delays the in vivo aging of skeletal muscle myofibers and progenitor cells. Aging (Albany NY). 2015;7:718-33.

27. Wu M, Desai DH, Kakarla SK, Katta A, Paturi S, Gutta AK, Rice KM, Walker Jr EM, Blough ER. Acetaminophen prevents aging-associated hyperglycemia in aged rats: effect of aging-associated hyperactivation of p38-MAPK and ERK1/2. Diabetes Metab Res Rev. 2009;25:279-86.

28. Perl A. mTOR activation is a biomarker and a central pathway to autoimmune disorders, cancer, obesity, and aging. Ann N Y Acad Sci. 2015; 1346:33-44.

29. Martins R, Lithgow GJ, Link W. Long live FOXO: unraveling the role of FOXO proteins in aging and longevity. Aging Cell. 2016;15:196-207.

30. Ekengren S, Hultmark D. A family of Turandot-related genes in the humoral stress response of Drosophila. Biochem Biophys Res Commun. 2001:284: 998-1003. 
31. Brun S, Vidal S, Spellman P, Takahashi K, Tricoire H, Lemaitre B. The MAPKKK Mekk1 regulates the expression of Turandot stress genes in response to septic injury in Drosophila. Genes Cells. 2006;11:397-407.

32. Kregel KC. Invited review: heat shock proteins: modifying factors in physiological stress responses and acquired thermotolerance. J Appl Physiol. 2002:92:2177-86.

33. Harman D. Aging: overview. Ann N Y Acad Sci. 2001;928:1-21.

34. Ikeyama S, Kokkonen G, Shack S, Wang X-T, Holbrook NJ. Loss in oxidative stress tolerance with aging linked to reduced extracellular signal-regulated kinase and Akt kinase activities. FASEB J. 2002;16:114-6.

35. Lin Y-J, Seroude L, Benzer S. Extended life-span and stress resistance in the Drosophila mutant methuselah. Science. 1998;282:943-6.

36. Guarente L, Kenyon C. Genetic pathways that regulate ageing in model organisms. Nature. 2000;408:255-62.

37. Kirkwood TB, Austad SN. Why do we age? Nature. 2000:408:233-8.

38. Ishii N, Fujii M, Hartman PS, Tsuda M, Yasuda K, Senoo-Matsuda N, Yanase S, Ayusawa D, Suzuki K. A mutation in succinate dehydrogenase cytochrome b causes oxidative stress and ageing in nematodes. Nature. 1998;394:694-7.

39. López-Otín C, Blasco MA, Partridge L, Serrano M, Kroemer G. The hallmarks of aging. Cell. 2013;153:1194-217.

40. Bahadorani S, Mukai S, Egli D, Hilliker AJ. Overexpression of metalresponsive transcription factor (MTF-1) in Drosophila melanogaster ameliorates life-span reductions associated with oxidative stress and metal toxicity. Neurobiol Aging. 2010;31:1215-26.

41. Coyle JT, Puttfarcken P. Oxidative stress, glutamate, and neurodegenerative disorders. Science. 1993;262:689-95.

42. Bharath S, Hsu M, Kaur D, Rajagopalan S, Andersen JK. Glutathione, iron and Parkinson's disease. Biochem Pharmacol. 2002;64:1037-48.

43. Toroser D, Sohal RS. Kinetic characteristics of native $\gamma$-glutamylcysteine ligase in the aging housefly, Musca domestica L. Biochem Biophys Res Comm. 2005;326:586-93.

\section{Submit your next manuscript to BioMed Central} and we will help you at every step:

- We accept pre-submission inquiries

- Our selector tool helps you to find the most relevant journal

- We provide round the clock customer support

- Convenient online submission

- Thorough peer review

- Inclusion in PubMed and all major indexing services

- Maximum visibility for your research

Submit your manuscript at www.biomedcentral.com/submit 